\title{
Association of arterial hemodynamics with left ventricular systolic function in hypertensive patients: A longitudinal study
}

\author{
Anna Teresa Goździk ${ }^{1, A, C-F}$, Ewelina Jasic-Szpak ${ }^{1, A, D-F}$, Jakub Michałowicz ${ }^{2, C, E, F}$, \\ Monika Przewłocka-Kosmala ${ }^{1, A, E, F}$, James Edward Sharman ${ }^{3, A B, B D-F}$, Wojciech Kosmala ${ }^{1, A-F}$ \\ ${ }^{1}$ Institute of Heart Diseases, Wroclaw Medical University, Poland \\ 2 Student Scientific Organization, Institute of Heart Diseases, Wroclaw Medical University, Poland \\ ${ }^{3}$ Menzies Institute for Medical Research, University of Tasmania, Hobart, Australia \\ A - research concept and design; $\mathrm{B}$ - collection and/or assembly of data; C - data analysis and interpretation; \\ $D$ - writing the article; $E$ - critical revision of the article; $F$ - final approval of the article
}

\section{Address for correspondence \\ Anna Teresa Goździk}

E-mail:anna.gozdzik@umed.wroc.pl

Funding sources

None declared

Conflict of interest

None declared

Received on July 10, 2021

Reviewed on July 27, 2021

Accepted on August 31, 2021

Published online on October 5, 2021

Cite as

Goździk AT, Jasic-Szpak E, Michałowicz J, PrzewłockaKosmala M, Sharman JE, Kosmala W. Association of arterial hemodynamics with left ventricular systolic function in hypertensive patients: A longitudinal study. Adv Clin Exp Med. 2022;30(11):1147-1156.

doi:10.17219/acem/141863

DOI

10.17219/acem/141863

Copyright

Copyright by Author(s)

This is an article distributed under the terms of the

Creative Commons Attribution 3.0 Unported (CC BY 3.0)

(https://creativecommons.org/licenses/by/3.0/)

\section{Abstract}

Background. Left ventricular (LV) systolic impairment, particularly in the longitudinal direction, is considered an early and sensitive marker of hypertensive heart disease and increased cardiovascular risk. The evidence indicates that aortic stiffness and central hemodynamic factors are important determinants of $L V$ performance, mediating the interaction between the heart and vascular load. Despite the existence of cross-sectional analyses linking central blood pressure (BP) parameters with LV mechanics, no longitudinal data are available which include serial measurements in the course of antihypertensive treatment.

Objectives. To investigate the associations between changes in LV longitudinal and circumferential function with alterations in arterial hemodynamics and ventricular-arterial coupling (VAC) in patients with uncomplicated hypertension during a 12-month follow-up.

Materials and methods. In this retrospective study, 216 patients (age $64.3 \pm 7.6$ years) underwent echocardiography including left ventricular longitudinal (GLS) and circumferential strain (GCS) analysis, brachial BP measurements, VAC (combining echocardiography and brachial BP), and arterial hemodynamics using radial tonometry at baseline and after 12 months of antihypertensive therapy. Patients were grouped into 2 subsets: with improvement in GLS $(n=103)$ and with deterioration in GLS $(n=113)$.

Results. No significant differences were observed in the majority of cardiovascular, demographic or clinical characteristics between the groups. The subset with improvement in GLS demonstrated more favorable changes over follow-up in pulse wave velocity $(p=0.03)$, central augmentation pressure $(p=0.01)$ and ventricular-arterial coupling $(p=0.04)$ compared to patients showing deterioration in GLS. In the multivariable analysis, independent determinants of changes in GLS were: GLS at baseline $(-0.48 ; p<0.001)$, changes from baseline to follow-up in central augmentation pressure $(-0.29 ; p=0.002)$ and ventriculararterial coupling $(-0.25 ; p=0.004)$. Independent determinants of analogous changes in GCS were: GCS at baseline $(-0.46 ; p<0.001)$ and changes in central augmentation pressure $(-0.22 ; p=0.02)$.

Conclusions. Left ventricular longitudinal and circumferential functional remodeling over time in hypertensive patients is associated with arterial hemodynamics and ventricular-arterial coupling.

Key words: hypertension, pulse wave analysis, arterial hemodynamics, ventricular-arterial coupling, longitudinal and circumferential strain 


\section{Background}

Left ventricular (LV) functional abnormalities have been widely recognized as an early manifestation of hypertensive heart disease (HHD) contributing to the increased cardiovascular risk. ${ }^{1-4}$ The introduction of new imaging techniques, particularly speckle tracking echocardiography, provided evidence that in addition to LV diastolic derangements, LV systolic impairment, especially in the longitudinal direction, should be considered an early and sensitive marker of LV functional compromise, resulting from the detrimental effect of hypertension (HT) ${ }^{5,6}$ Changes in LV circumferential contractility in the natural history of disease processes are not well understood; however, the relatively delayed development of this dysfunction has been reported consistently. ${ }^{7-11}$

Accumulating evidence indicates that aortic stiffness and central hemodynamic factors are important determinants of LV performance, mediating the interaction between the heart and vascular load. ${ }^{12}$ Specifically, LV pressure load and myocardial function are associated with hemodynamics in the proximal aorta. Increased aortic stiffness amplifies early systolic load (evidenced by an increase in central pressure augmentation) and impairs ventricular-arterial coupling (VAC), which adversely affects cardiac function. Moreover, alterations in LV and aortic physiology enhance the risk of heart failure, especially heart failure with preserved ejection fraction (HFpEF), thus paving the way for adverse outcomes, such as $\mathrm{HT}^{13}$

The association between central loading factors, assessed noninvasively with pulse wave analysis (PWA) from peripheral (e.g. brachial) arteries, and LV performance and structure has been demonstrated in previous studies. ${ }^{14-21}$ However, no diagnostic approach has been developed that would favor the use of specific central hemodynamic parameters. Moreover, to our knowledge, no longitudinal data are available concerning the correlation of central hemodynamic parameters, including VAC, with LV mechanics, in the course of antihypertensive treatment.

\section{Objectives}

The aim of this study was to investigate the relationship between alterations in LV longitudinal and circumferential function with changes in arterial hemodynamic characteristics and VAC in patients with uncomplicated HT during a 12-month follow-up.

\section{Materials and methods}

\section{Study subjects}

This study was a retrospective analysis of 216 patients with uncomplicated HT, including 105 men and 111 women who participated in the BP GUIDE study, ${ }^{22}$ and had complete datasets including LV deformation and PWA parameters. Among inclusion criteria were: 1875 years of age, lack of pregnancy, and antihypertensive therapy including from 1 to 3 hypotensive drugs. Exclusion criteria included: severely abnormal LV mass index $\left(>59 \mathrm{~g} / \mathrm{m}^{2.7}\right.$ in women and $>64 \mathrm{~g} / \mathrm{m}^{2.7}$ in men), clinical history of coronary artery disease or renal disease, serum creatinine $>1.6 \mathrm{mg} / \mathrm{dL}$, secondary HT, uncontrolled HT (office brachial blood pressure (BP) $>180 / 100 \mathrm{~mm} \mathrm{Hg}$ ), aortic valve stenosis, or upper limb obstructive atherosclerosis. All patients were in the sinus rhythm to ensure the reliability of myocardial deformation assessment and PWA.

\section{Protocol}

Each participant underwent echocardiography, including LV longitudinal and circumferential strain analysis, brachial BP measurements, and estimated central hemodynamics assessment using radial tonometry at baseline and after 12 months of supervised HT management. Medication quantity was determined by daily defined dose (DDD) and according to the World Health Organization standards for exact quantifications of drug amount and standardization. ${ }^{23}$ Patients were stratified according to the changes in global longitudinal strain (GLS) over the follow-up period (follow-up value minus baseline value) into 2 groups: with improvement in GLS and with deterioration in GLS. The study was conducted in accordance with The Declaration of Helsinki, and the protocol was approved by the institutional bioethics committee. All subjects gave their informed consent for inclusion before they participated in the study.

\section{Echocardiography}

Echocardiographic imaging was performed using Vivid E9 and Vivid 7 equipment (GE; Vingmed Ultrasound AS, Horten, Norway) with phased array $2.5 \mathrm{MHz}$ multifrequency transducers. The assessment of LV function at baseline and 12-month follow-up was carried out by a single expert observer (W.K.) who was blinded to the clinical data.

\section{Conventional and tissue Doppler imaging}

The measurements of cardiac dimensions, wall thicknesses, LV ejection fraction, and Doppler parameters of mitral inflow and mitral annular velocities were obtained according to the recommendations of the American Society of Echocardiography and the European Association of Cardiovascular Imaging. ${ }^{24}$

The assessment of LV mass and ejection fraction were calculated by real-time three-dimensional echocardiography. The left atrium (LA) volume was measured using Simpson's technique from the apical four-chamber and two-chamber views at the end of ventricular systole, just 
before mitral valve opening. The LA volume index was computed by adjusting LA volume to body surface area. The LV inflow parameters were evaluated using pulsed wave Doppler from the apical four-chamber view with the sample volume placed between the tips of mitral leaflets, and included peak early (E) and late diastolic flow velocity (A), along with deceleration time of the E-wave (DT). Pulse wave tissue Doppler was used to define peak early diastolic tissue velocity $\left(\mathrm{e}^{\prime}\right)$ at the septal and lateral parts of the mitral annulus. The ratio of mitral inflow early diastolic velocity to the average $\mathrm{e}^{\prime}$ velocity obtained from the septal and lateral sides of the mitral annulus (E/e') was calculated to estimate LV filling pressure. ${ }^{25}$

\section{Speckle tracking imaging}

Left ventricular deformation was assessed using a semiautomated two-dimensional speckle tracking technique (EchoPac; General Electric Healthcare, Horten, Norway) that analyzed all segments of the left ventricle in the 3 apical views (longitudinal strain) and parasternal short axis view at the papillary muscle level (circumferential strain). The LV strain measurements were performed at a frame rate of 60-80 frames/s. The measurements included the greatest negative value on the strain curve. Global longitudinal (GLS) and circumferential strains (GCS) were calculated as the averages of all LV segments interrogated. If more than 2 segments in a single view remained poorly tracked despite manual correction of the region of interest, global deformation parameters were not computed. All echocardiographic parameters were averaged over 3 consecutive cardiac cycles and reported as absolute values.

\section{BP and hemodynamic measurements}

Office brachial BP was measured in duplicate after 10 min of rest in the sitting position using a validated device (Omron HEM 907; Omron Healthcare, Kyoto, Japan). Central hemodynamics were estimated using radial tonometry (SphygmoCor; AtCor Medical, Sydney, Australia) with calibration of radial waveforms, using brachial systolic blood pressure (SBP) and diastolic blood pressure (DBP). This approach seeks to preserve the level of difference in SBP between brachial and central arterial sites, but underestimates the true central aortic SBP. ${ }^{26}$ Hence, the focus of this analysis was on waveform indices, including augmented pressure and augmentation index from both, the untransformed radial waveform as well as the derived central waveform. Augmented pressure was assessed as the pressure difference between the $1^{\text {st }}$ and $2^{\text {nd }}$ systolic peaks on the pressure waveforms. Augmentation index was calculated as a percentage of augmented pressure to pulse pressure on the central and radial waveforms. To correct for differences in heart rate (HR), augmented pressure and augmentation index values were adjusted to a standard of $75 \mathrm{bpm}$. Pulse pressure was computed by the difference between SBP and DBP at brachial and central sites. Pulse pressure amplification was calculated as the ratio of brachial to central pulse pressure. Twenty-four-hour ambulatory BP was recorded using TM 2430 equipment (A\&D Mercury, Thebarton, Australia).

\section{Combined BP-echocardiography variables}

The ventricular-arterial coupling ratio was calculated as the quotient of aortic (EaI) and LV end-systolic elastance (ELVI) indices, where EaI and ELVI were defined as the ratio of end-systolic pressure to echocardiographicderived stroke volume index and end-systolic volume index, respectively. End-systolic pressure was computed from the equation $0.9 \times$ brachial SBP. ${ }^{27}$ Peripheral vascular resistance index was calculated by mean arterial pressure/ cardiac index (the product of two-dimensional stroke volume and HR indexed to body surface area).

\section{Statistical analyses}

Data are presented as mean values \pm standard deviation (SD) for continuous variables and as counts and percentages for categorical variables. Homogeneity of variances was assessed using the Levene's test. Between groups, comparisons were performed using an unpaired two-sided Students $\mathrm{t}$-test for continuous variables and $X^{2}$ test for categorical variables. The associations of changes in GLS and GCS from baseline to follow-up with other variables were analyzed using Pearson's correlation coefficient and multivariable linear regression analysis. The components of multivariable models were selected on the basis of anticipated and demonstrated univariate associations. Changes in particular parameters were calculated by subtracting the baseline value from the follow-up value, and were expressed in the units of their measurements. The reproducibility of strain measurements was evaluated by the Bland-Altman method (mean difference and 95\% confidence interval (95\% CI)). All calculations were carried out with standard statistical software (STATISTICA v. 13; StatSoft Inc., Tulsa, USA). The level of statistical significance was set at a twosided p-value $<0.05$.

\section{Results}

Patients were grouped into 2 subsets depending on the change in the value of GLS after 12 months of antihypertensive therapy: with improvement in GLS ( $\mathrm{n}=103)$ and with deterioration in GLS ( $\mathrm{n}=113)$.

\section{Patient profile}

Baseline demographic, clinical and cardiovascular characteristics according to the change in GLS are presented in Table 1. A significantly lower GLS at baseline was found 
Table 1. Baseline demographic, clinical and cardiovascular characteristics according to the change in global longitudinal strain over a 12-month follow-up

\begin{tabular}{|c|c|c|c|}
\hline Variables & $\begin{array}{l}\text { GLS deterioration } \\
\text { at follow-up } \\
n=113\end{array}$ & $\begin{array}{l}\text { GLS improvement } \\
\text { at follow-up } \\
n=103\end{array}$ & $p$-value \\
\hline Age [years] & $64.9 \pm 7.2$ & $63.7 \pm 8.0$ & 0.23 \\
\hline Male sex, n (\%) & $51(45)$ & $52(50)$ & 0.60 \\
\hline BMI $\left[\mathrm{kg} / \mathrm{m}^{2}\right]$ & $29.4 \pm 4.6$ & $29.7 \pm 5.1$ & 0.55 \\
\hline Creatinine $[\mathrm{mg} / \mathrm{dL}]$ & $0.88 \pm 0.18$ & $0.88 \pm 0.18$ & 0.96 \\
\hline $\operatorname{eGFR}\left[\mathrm{mL} / \mathrm{min} / 1.73 \mathrm{~m}^{2}\right]$ & $82.1 \pm 14.8$ & $84.1 \pm 15.1$ & 0.33 \\
\hline Mean 24-hour SBP [mm Hg] & $131.1 \pm 11.5$ & $134.1 \pm 12.7$ & 0.073 \\
\hline Mean 24-hour DBP [mm Hg] & $75.5 \pm 7.5$ & $77.1 \pm 7.6$ & 0.13 \\
\hline bSBP $[\mathrm{mm} \mathrm{Hg}]$ & $132.9 \pm 14.9$ & $130.6 \pm 12.9$ & 0.24 \\
\hline $\mathrm{bDBP}[\mathrm{mm} \mathrm{Hg}]$ & $76.9 \pm 9.2$ & $77.4 \pm 9.8$ & 0.66 \\
\hline bPP [mm Hg] & $56.0 \pm 11.8$ & $53.1 \pm 10.9$ & 0.07 \\
\hline $\mathrm{PWV}[\mathrm{m} / \mathrm{s}]$ & $9.5 \pm 2.2$ & $9.4 \pm 2.1$ & 0.59 \\
\hline cAGPH HR 75 [\%] & $21 \pm 8.7$ & $21 \pm 9.7$ & 0.87 \\
\hline CAP HR 75 [mm Hg] & $9.0 \pm 4.9$ & $8.9 \pm 5.1$ & 0.92 \\
\hline rAlx [\%] & $81 \pm 13$ & $82 \pm 16$ & 0.64 \\
\hline PVRI [U/m²] & $53.5 \pm 23.4$ & $54.3 \pm 14.4$ & 0.76 \\
\hline Eai & $4.2 \pm 1.9$ & $4.3 \pm 1.0$ & 0.57 \\
\hline ELVi & $8.7 \pm 3.0$ & $8.2 \pm 2.5$ & 0.22 \\
\hline VAC & $0.51 \pm 0.27$ & $0.55 \pm 0.15$ & 0.13 \\
\hline LV diastolic diameter [cm] & $4.4 \pm 0.5$ & $4.3 \pm 0.4$ & 0.31 \\
\hline LV mass index $\left[\mathrm{g} / \mathrm{m}^{2}\right]$ & $30.9 \pm 5.3$ & $31.3 \pm 5.5$ & 0.56 \\
\hline LA volume index $\left[\mathrm{mL} / \mathrm{m}^{2}\right]$ & $32.9 \pm 9.7$ & $33.5 \pm 11.3$ & 0.72 \\
\hline LV ejection fraction [\%] & $62.2 \pm 4.9$ & $60.8 \pm 5.7$ & 0.072 \\
\hline GLS [\%] & $19.4 \pm 2.1$ & $17.7 \pm 2.1$ & $<0.001$ \\
\hline GCS [\%] & $17.3 \pm 2.8$ & $17.1 \pm 2.8$ & 0.64 \\
\hline$E / A$ & $0.99 \pm 0.3$ & $0.96 \pm 0.2$ & 0.32 \\
\hline DT [ms] & $226.9 \pm 44.5$ & $221.7 \pm 48.8$ & 0.42 \\
\hline e' septal [cm/s] & $6.3 \pm 1.5$ & $6.1 \pm 1.4$ & 0.35 \\
\hline $\mathrm{e}^{\prime}$ lateral $[\mathrm{cm} / \mathrm{s}]$ & $8.3 \pm 1.9$ & $8.0 \pm 2.0$ & 0.34 \\
\hline$E / e^{\prime}$ & $10.0 \pm 3.1$ & $9.6 \pm 2.7$ & 0.37 \\
\hline DDD & $2.5 \pm 1.5$ & $2.4 \pm 1.4$ & 0.45 \\
\hline ACEi, n (\%) & $35(31)$ & $30(29)$ & 0.77 \\
\hline ARB, n (\%) & $72(64)$ & $68(66)$ & 0.72 \\
\hline CCB, n (\%) & $33(29)$ & $35(34)$ & 0.45 \\
\hline Diuretics, n (\%) & $44(39)$ & $39(38)$ & 0.87 \\
\hline$\beta$-blockers, n (\%) & $11(10)$ & $11(11)$ & 0.82 \\
\hline
\end{tabular}

GLS - global longitudinal strain; BMI - body mass index; eGFR - estimated glomerular filtration rate; SBP - systolic blood pressure; DBP - diastolic blood pressure; bSBP - brachial systolic blood pressure; bDBP - brachial diastolic blood pressure; bPP - brachial pulse pressure; PWV - pulse wave velocity; CAGPH HR 75 - central augmentation index at heart rate 75 beats/min; CAP HR 75 - central augmentation pressure at heart rate 75 beats/min; PVRI - peripheral vascular resistance index; Eai - effective arterial elastance index; ELVi - left ventricular elastance index; VAC - ventricular-arterial coupling; LV - left ventricular; LA - left atrium; GCS - global circumferential strain; E/A - ratio of peak early to peak late diastolic mitral inflow velocity; DT - deceleration time; $e^{\prime}$ - peak early diastolic mitral annular velocity; E/e' - ratio of peak early diastolic mitral inflow velocity to peak early diastolic mitral annular velocity; rAlx - radial augmentation index; DDD - daily defined dose of hypotensive drugs; ACEi - angiotensin-converting enzyme inhibitors; ARB - angiotensin receptor blockers; CCB - calcium channel blockers. The eGFR was calculated according to the CKD-EPI formula (www.nice.org.uk (July 2014)) "Chronic kidney disease in adults: assessment and management". Values in bold are statistically significant. in patients with the improvement in GLS at follow-up. There were no significant integroup differences in patient age, proportion of male sex, body mass index (BMI), daily defined dose (DDD) of antihypertensive drugs, as well as echocardiographic, BP, or hemodynamic measurements.

\section{Blood pressure and cardiovascular characteristics}

The subset with improvement in GLS demonstrated more favorable changes over followup in pulse wave velocity, central augmentation pressure at HR 75 beats/min, VAC, GCS, and septal peak early diastolic mitral annular velocity compared to patients with deterioration in GLS (Table 2).

\section{Correlates of LV functional characteristics}

Univariate associations of changes in GLS and GCS from baseline to follow-up, with baseline values of demographic, clinical and cardiovascular characteristics are presented in Table 3 , and analogous associations with changes from baseline to follow-up in clinical and cardiovascular parameters are shown in Table 4. Among significant correlates of change in GLS at 12 months were pulse wave velocity, VAC, effective arterial elastance index, central augmentation pressure, and index at $\mathrm{HR} 75$ beats/min, whereas significant correlates of change in GCS were DBP, central augmentation pressure and index at $\mathrm{HR}$ of 75 beats/min.

Multivariable linear regression models illustrated that the independent determinants of changes in GLS at a follow-up were GLS at baseline and changes over follow-up in central augmentation pressure and VAC, whereas the independent determinants of changes in GCS were GCS at baseline and changes over follow-up in central augmentation pressure or index (Table 5). Changes over follow-up in central augmentation pressure and index were not combined into a single multivariable model due to strong collinearity $(\mathrm{r}=0.85)$. Similar but not statistically significant results were demonstrated for radial augmentation index when used instead of central augmentation parameters (Table 6).

Change in effective arterial elastance index over follow-up was tested in multivariable models instead of change in VAC (a very strong collinearity between these 2 variables with $r=0.95)$, but did not prove to be a significant determinant of either delta GLS ( $p=0.069)$ or delta GCS $(\mathrm{p}=0.71)$. 
Table 2. Changes in clinical and cardiovascular characteristics according to changes in global longitudinal strain over a 12-month follow-up

\begin{tabular}{|c|c|c|c|}
\hline Variables & $\begin{array}{l}\text { GLS deterioration } \\
\text { at follow-up } \\
n=113\end{array}$ & $\begin{array}{l}\text { GLS improvement } \\
\text { at follow-up } \\
n=103\end{array}$ & p-value \\
\hline$\triangle \mathrm{DDD}$ & $-0.36 \pm 0.95$ & $-0.19 \pm 0.93$ & 0.20 \\
\hline$\triangle$ Mean 24-hour SBP [mm Hg] & $4.1 \pm 10.5$ & $1.9 \pm 12.9$ & 0.18 \\
\hline$\triangle$ Mean 24-hour DBP [mm Hg] & $1.4 \pm 6.1$ & $1.2 \pm 8$ & 0.90 \\
\hline$\triangle \mathrm{bSBP}[\mathrm{mm} \mathrm{Hg}]$ & $-1.2 \pm 15.9$ & $-1.3 \pm 12.3$ & 0.96 \\
\hline$\triangle \mathrm{bDBP}[\mathrm{mm} \mathrm{Hg}]$ & $0.05 \pm 9.0$ & $-0.7 \pm 8.9$ & 0.54 \\
\hline$\triangle \mathrm{bPP}[\mathrm{mm} \mathrm{Hg}]$ & $-1.3 \pm 10.8$ & $-0.6 \pm 8.2$ & 0.62 \\
\hline$\triangle \mathrm{PWV}[\mathrm{m} / \mathrm{s}]$ & $0.3 \pm 1.6$ & $-0.3 \pm 1.9$ & 0.026 \\
\hline$\triangle \mathrm{CAGPH} H \mathrm{HR} 75[\%]$ & $1.2 \pm 7.5$ & $-0.5 \pm 6.3$ & 0.09 \\
\hline$\triangle \mathrm{CAP} H \mathrm{R} 75[\mathrm{~mm} \mathrm{Hg}]$ & $0.7 \pm 4.5$ & $-0.7 \pm 3.4$ & 0.012 \\
\hline$\triangle \mathrm{rAlx}[\%]$ & $1.4 \pm 12.7$ & $-1.2 \pm 10.5$ & 0.11 \\
\hline$\triangle P V R I\left[U / m^{2}\right]$ & $4.4 \pm 24.3$ & $-1 \pm 28.2$ & 0.15 \\
\hline$\triangle$ Eai & $0.3 \pm 1.7$ & $-0.1 \pm 1.7$ & 0.079 \\
\hline$\triangle E L V i$ & $0.1 \pm 1.1$ & $-0.1 \pm 0.8$ & 0.29 \\
\hline$\triangle \mathrm{VAC}$ & $0.05 \pm 0.24$ & $-0.01 \pm 0.20$ & 0.049 \\
\hline$\Delta$ LV diastolic diameter $[\mathrm{cm}]$ & $-0.06 \pm 0.4$ & $0.004 \pm 0.5$ & 0.33 \\
\hline$\Delta \mathrm{LV}$ mass index $\left[\mathrm{g} / \mathrm{m}^{2}\right]$ & $0.07 \pm 2.6$ & $-0.25 \pm 2.0$ & 0.43 \\
\hline$\Delta \mathrm{LA}$ volume index $\left[\mathrm{mL} / \mathrm{m}^{2}\right]$ & $-0.48 \pm 7.1$ & $0.32 \pm 9.2$ & 0.48 \\
\hline$\Delta$ LV ejection fraction $[\%]$ & $-0.05 \pm 5.3$ & $1.3 \pm 5.8$ & 0.085 \\
\hline$\triangle \mathrm{GLS}[\%]$ & $-1.4 \pm 1.2$ & $1.5 \pm 1.0$ & $<0.001$ \\
\hline$\triangle \mathrm{GCS}[\%]$ & $-0.2 \pm 2.8$ & $1.1 \pm 2.5$ & $<0.001$ \\
\hline$\triangle E / A$ & $-0.02 \pm 0.27$ & $0.02 \pm 0.22$ & 0.28 \\
\hline$\triangle \mathrm{DT}[\mathrm{ms}]$ & $7.2 \pm 52.9$ & $5.0 \pm 55.6$ & 0.78 \\
\hline$\Delta e^{\prime}$ septal $[\mathrm{cm} / \mathrm{s}]$ & $-0.4 \pm 1.3$ & $0.09 \pm 1.3$ & 0.005 \\
\hline$\Delta \mathrm{e}^{\prime}$ lateral $[\mathrm{cm} / \mathrm{s}]$ & $-0.6 \pm 1.5$ & $-0.2 \pm 1.8$ & 0.094 \\
\hline$\Delta \mathrm{E} / \mathrm{e}^{\prime}$ & $0.5 \pm 2.3$ & $0.3 \pm 2.8$ & 0.56 \\
\hline
\end{tabular}

$\triangle$ - change at 12-month follow-up (follow-up value minus baseline value); DDD - daily defined dose of hypotensive drugs; SBP - systolic blood pressure; DBP - diastolic blood pressure; bSBP - brachial systolic blood pressure; bDBP - brachial diastolic blood pressure; bPP - brachial pulse pressure; PWV - pulse wave velocity; CAGPH HR 75 - central augmentation index at heart rate 75 beats/min; CAP HR 75 - central augmentation pressure at heart rate 75 beats/min; Eai - effective arterial elastance index; PVRI - peripheral vascular resistance index; LV - left ventricular; LA - left atrial; GLS - global longitudinal strain; GCS - global circumferential strain; VAC - ventricular-arterial coupling; E/A - ratio of peak early to peak late diastolic mitral inflow velocity; DT - deceleration time; e' - peak early diastolic mitral annular velocity; E/e' - ratio of peak early diastolic mitral inflow velocity to peak early diastolic mitral annular velocity; rAlx - radial augmentation index. Values in bold are statistically significant.
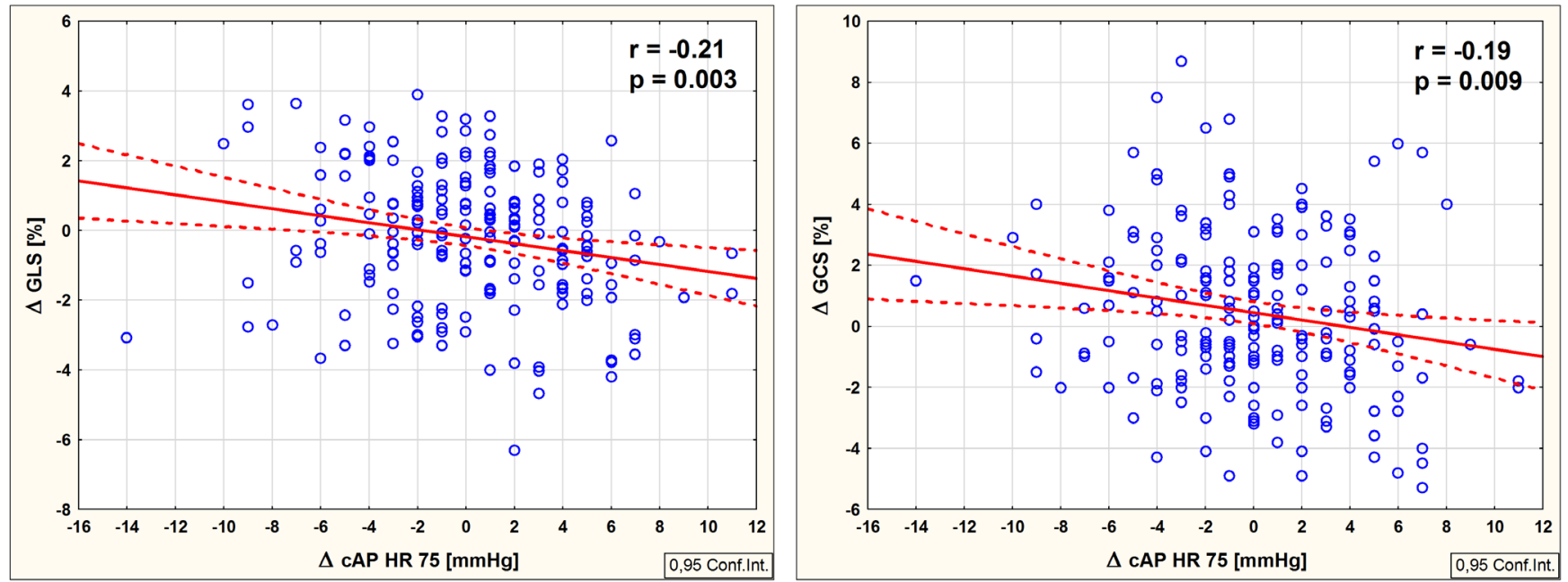

Fig. 1. Associations between changes in central augmentation pressure and changes in left ventricular global longitudinal and circumferential deformation over a 12-month follow-up. CAP HR 75 - central augmentation pressure at heart rate 75 beats/min; GLS - global longitudinal strain; GCS - global circumferential strain 
Table 3. Univariable associations of changes in global longitudinal strain and circumferential strain at a 12-month follow-up with demographic, clinical and cardiovascular characteristics at baseline

\begin{tabular}{|c|c|c|c|c|}
\hline \multirow{2}{*}{ Variables } & \multicolumn{2}{|c|}{$\Delta \mathrm{GLS}$} & \multicolumn{2}{|c|}{$\triangle \mathrm{GCS}$} \\
\hline & $r$ & $p$-value & $r$ & $p$-value \\
\hline Age [years] & -0.01 & 0.90 & 0.01 & 0.83 \\
\hline BMI $\left[\mathrm{kg} / \mathrm{m}^{2}\right]$ & 0.03 & 0.62 & 0.01 & 0.97 \\
\hline DDD & -0.05 & 0.42 & -0.03 & 0.69 \\
\hline Mean 24-hour SBP [mm Hg] & 0.08 & 0.22 & 0.19 & 0.007 \\
\hline Mean 24-hour DBP [mm Hg] & 0.07 & 0.31 & 0.18 & 0.009 \\
\hline $\mathrm{bSBP}[\mathrm{mm} \mathrm{Hg}]$ & -0.04 & 0.57 & 0.04 & 0.58 \\
\hline $\mathrm{bDBP}[\mathrm{mm} \mathrm{Hg}]$ & 0.05 & 0.44 & 0.07 & 0.34 \\
\hline $\mathrm{bPP}[\mathrm{mm} \mathrm{Hg}]$ & -0.09 & 0.18 & -0.01 & 0.90 \\
\hline $\mathrm{PWV}[\mathrm{m} / \mathrm{s}]$ & 0.01 & 0.82 & 0.01 & 0.90 \\
\hline CAGPH HR 75 [\%] & 0.04 & 0.51 & -0.04 & 0.55 \\
\hline CAP HR 75 [mm Hg] & 0.02 & 0.77 & -0.01 & 0.85 \\
\hline rAlx [\%] & 0.08 & 0.26 & -0.05 & 0.50 \\
\hline PVRI [U/m²] & 0.08 & 0.24 & 0.02 & 0.82 \\
\hline Eai & 0.09 & 0.20 & 0.05 & 0.48 \\
\hline ELVi & -0.10 & 0.15 & -0.13 & 0.077 \\
\hline VAC & 0.17 & 0.016 & 0.15 & 0.035 \\
\hline LV diastolic diameter [cm] & -0.07 & 0.33 & 0.13 & 0.076 \\
\hline LV mass index $\left[\mathrm{g} / \mathrm{m}^{2}\right]$ & 0.03 & 0.69 & 0.05 & 0.48 \\
\hline $\mathrm{LA}$ volume index $\left[\mathrm{mL} / \mathrm{m}^{2}\right]$ & -0.03 & 0.69 & 0.09 & 0.19 \\
\hline LV ejection fraction [\%] & -0.13 & 0.078 & -0.12 & 0.10 \\
\hline GLS [\%] & -0.48 & $<0.001$ & -0.16 & 0.027 \\
\hline GCS [\%] & -0.08 & 0.27 & -0.46 & $<0.001$ \\
\hline
\end{tabular}

BMI - body mass index; DDD - daily defined dose of hypotensive drugs; SBP - systolic blood pressure; DBP - diastolic blood pressure; bSBP - brachial systolic blood pressure; bDBP - brachial diastolic blood pressure; bPP - brachial pulse pressure; PWV - pulse wave velocity; cAGPH HR 75 - central augmentation index at heart rate 75 beats/min; CAP HR 75 - central augmentation pressure at heart rate 75 beats/min; PVRI - peripheral vascular resistance index; Eai - effective arterial elastance index; ELVi - left ventricular elastance index; VAC - ventricular-arterial coupling; LV - left ventricular; LA - left atrium; GLS - global longitudinal strain; GCS - global circumferential strain; rAlx - radial augmentation index. Values in bold are statistically significant.

Scatterplots illustrating associations between changes in central augmentation pressure at a $\mathrm{HR}$ of 75 beats/min and LV longitudinal and circumferential function are presented in Fig. 1.

\section{Sensitivity analysis}

To check the robustness of results, we changed the input variables by restricting the dataset to the upper and lower delta GLS tertiles. This analysis confirmed the relationships between multivariable predictors (for example, the changes over follow-up in central augmentation pressure and index, or VAC), and changes in GLS and GCS (Table 7).

\section{Reproducibility}

The intra-observer variability of myocardial deformation measurements was assessed in 15 randomly selected examinations, and was analyzed twice on 2 separate days within a four-week time interval. The variability was $-0.3 \%$
$(-0.7 ; 0.2)$ for the LV longitudinal strain and $0.5 \%(-0.5 ; 1.4)$ for the circumferential strain.

\section{Discussion}

The current study confirmed the link between LV function and central hemodynamic and aortic characteristics by showing independent associations between temporal and treatment-associated changes in GLS, and changes in central augmentation pressure and VAC, as well as between analogous changes in GCS and central augmentation pressure. Thus, our findings emphasize the role of central loading factors in the pathophysiology of HHD, and support the need for extending BP assessments beyond standard cuff measurements to effectively manage myocardial complications of HT.

Existing evidence indicates the importance of both central and peripheral BP measurements due to their associations with cardiac, renal and vascular injury. Recognition of target organ damage is essential for clinical 
Table 4. Univariable associations of changes in global longitudinal strain and circumferential strain with changes in clinical and cardiovascular characteristics at a 12-month follow-up

\begin{tabular}{|c|c|c|c|c|}
\hline \multirow{2}{*}{ Variables } & \multicolumn{2}{|c|}{$\Delta \mathrm{GLS}$} & \multicolumn{2}{|c|}{$\triangle \mathrm{GCS}$} \\
\hline & r & $p$-value & $r$ & $p$-value \\
\hline$\triangle \mathrm{DDD}$ & 0.10 & 0.16 & 0.11 & 0.15 \\
\hline$\triangle$ Mean 24-hour SBP [mm Hg] & -0.07 & 0.34 & 0.04 & 0.63 \\
\hline$\triangle$ Mean 24-hour DBP [mm Hg] & -0.01 & 0.93 & 0.01 & 0.88 \\
\hline$\triangle \mathrm{bSBP}[\mathrm{mm} \mathrm{Hg}]$ & -0.07 & 0.30 & -0.09 & 0.24 \\
\hline$\triangle \mathrm{bDBP}[\mathrm{mm} \mathrm{Hg}]$ & -0.12 & 0.094 & -0.15 & 0.039 \\
\hline$\triangle \mathrm{bPP}[\mathrm{mm} \mathrm{Hg}]$ & 0.02 & 0.98 & 0.01 & 0.94 \\
\hline$\triangle P W V$ & -0.17 & 0.015 & 0.05 & 0.52 \\
\hline$\triangle \mathrm{cAGPH} H \mathrm{HR} 75[\%]$ & -0.15 & 0.034 & -0.18 & 0.013 \\
\hline$\triangle \mathrm{cAP} H \mathrm{R} 75[\mathrm{~mm} \mathrm{Hg}]$ & -0.21 & 0.003 & -0.19 & 0.009 \\
\hline$\Delta \mathrm{rAlx}[\%]$ & -0.13 & 0.063 & -0.14 & 0.051 \\
\hline$\triangle P V R I\left[U / m^{2}\right]$ & -0.13 & 0.059 & 0.01 & 0.99 \\
\hline$\triangle$ Eai & -0.17 & 0.011 & -0.04 & 0.60 \\
\hline$\triangle E L V i$ & 0.09 & 0.20 & -0.01 & 0.87 \\
\hline$\triangle \mathrm{VAC}$ & -0.20 & 0.003 & -0.02 & 0.83 \\
\hline$\Delta \mathrm{LV}$ diastolic diameter $[\mathrm{cm}]$ & 0.12 & 0.094 & 0.04 & 0.56 \\
\hline$\Delta \mathrm{LV}$ mass index $\left[\mathrm{g} / \mathrm{m}^{2}\right]$ & -0.01 & 0.84 & 0.02 & 0.74 \\
\hline$\Delta \mathrm{LA}$ volume index $\left[\mathrm{mL} / \mathrm{m}^{2}\right]$ & 0.06 & 0.40 & 0.02 & 0.83 \\
\hline$\Delta$ LV ejection fraction [\%] & 0.13 & 0.072 & 0.02 & 0.77 \\
\hline
\end{tabular}

$\triangle$ - change at 12-month follow-up (follow-up value minus baseline value); DDD - daily defined dose of hypotensive drugs; SBP - systolic blood pressure; DBP - diastolic blood pressure; bSBP - brachial systolic blood pressure; bDBP - brachial diastolic blood pressure; bPP - brachial pulse pressure; PWV - pulse wave velocity; CAGPH HR 75 - central augmentation index at heart rate 75 beats/min; CAP HR 75 - central augmentation pressure at heart rate 75 beats/min; PVRI - peripheral vascular resistance index; Eai - effective arterial elastance index; LV - left ventricular; LA - left atrial; GLS - global longitudinal strain; VAC - ventricular-arterial coupling; rAlx - radial augmentation index. Values in bold are statistically significant.

Table 5. Multivariable associations of the change in global longitudinal and circumferential strains at a 12-month follow-up. Models with central augmentation parameters

\begin{tabular}{|c|c|c|c|c|c|c|c|c|c|c|c|c|}
\hline \multirow[t]{2}{*}{ Variables } & \multicolumn{3}{|c|}{$\begin{array}{c}\text { Model } 1 \\
\Delta \mathrm{GLS} \\
\mathrm{R}^{2}=0.29\end{array}$} & \multicolumn{3}{|c|}{$\begin{array}{c}\text { Model } 2 \\
\Delta \mathrm{GLS} \\
\mathrm{R}^{2}=0.28\end{array}$} & \multicolumn{3}{|c|}{$\begin{array}{c}\text { Model } 3 \\
\triangle \mathrm{GCS} \\
\mathrm{R}^{2}=0.25\end{array}$} & \multicolumn{3}{|c|}{$\begin{array}{c}\text { Model } 4 \\
\begin{array}{c}\Delta \mathrm{GCS} \\
\mathrm{R}^{2}=0.26\end{array}\end{array}$} \\
\hline & $\beta$ & SE & $p$-value & $\beta$ & SE & $p$-value & $\beta$ & SE & $\mathrm{p}$-value & $\beta$ & SE & $p$-value \\
\hline GLS at baseline & -0.45 & 0.07 & $<0.001$ & -0.46 & 0.07 & $<0.001$ & - & - & - & - & - & - \\
\hline GCS at baseline & - & - & - & - & - & - & -0.45 & 0.07 & $<0.001$ & -0.47 & 0.07 & $<0.001$ \\
\hline$\triangle \mathrm{CAP} H \mathrm{R} 75$ & -0.17 & 0.07 & 0.016 & - & - & - & -0.16 & 0.07 & 0.024 & - & - & - \\
\hline$\triangle \mathrm{CAGPH} H \mathrm{HR} 75$ & - & - & - & -0.13 & 0.07 & 0.051 & - & - & - & -0.19 & 0.07 & 0.006 \\
\hline$\triangle \mathrm{VAC}$ & -0.16 & 0.07 & 0.014 & -0.15 & 0.07 & 0.019 & 0.03 & 0.07 & 0.68 & 0.04 & 0.07 & 0.59 \\
\hline$\triangle P W V$ & -0.07 & 0.07 & 0.27 & -0.09 & 0.07 & 0.18 & 0.11 & 0.07 & 0.14 & 0.10 & 0.07 & 0.17 \\
\hline$\triangle$ Mean 24-hour SBP & 0.03 & 0.07 & 0.62 & 0.02 & 0.07 & 0.76 & 0.04 & 0.07 & 0.63 & 0.03 & 0.07 & 0.71 \\
\hline$\triangle \mathrm{DDD}$ & -0.01 & 0.07 & 0.83 & 0.01 & 0.07 & 0.91 & 0.04 & 0.07 & 0.60 & 0.03 & 0.07 & 0.64 \\
\hline
\end{tabular}

$\triangle$ - change at 12-month follow-up (follow-up value minus baseline value); GLS - global longitudinal strain; GCS - global circumferential strain; CAP HR 75 - central augmentation pressure at heart rate 75 beats/min; CAGPH HR 75 - central augmentation index at heart rate 75 beats/min; VAC - ventricular-arterial coupling; PWV - pulse wave velocity; SBP - systolic blood pressure; DDD - daily defined dose of hypertension drugs; SE - standard error. Values in bold are statistically significant.

risk stratification in hypertensive patients, and reducing the occurrence and severity of these complications represents a major goal of current therapy. Central BP and associated indices including augmented pressure, central pulse pressure, and effective arterial elastance index have been found to be more closely related to the target organ damage expressed as LV hypertrophy, cardiac dysfunction, and increased carotid intima media thickness, and are believed to reflect LV afterload more accurately than brachial BP measurements. ${ }^{28-31}$ Nonetheless, derived measures of central augmentation index are highly correlated with peripheral (radial) augmentation index ${ }^{32}$ (e.g., 
Table 6. Multivariable associations of the change in global longitudinal and circumferential strains at a 12-month follow-up. Models with radial augmentation index

\begin{tabular}{|c|c|c|c|c|c|c|}
\hline \multirow[t]{2}{*}{ Variables } & \multicolumn{3}{|c|}{$\begin{array}{c}\text { Model } 1 \\
\Delta \mathrm{GLS} \\
\mathrm{R}^{2}=0.28\end{array}$} & \multicolumn{3}{|c|}{$\begin{array}{c}\text { Model } 2 \\
\triangle \mathrm{GCS} \\
\mathrm{R}^{2}=0.24\end{array}$} \\
\hline & $\beta$ & SE & $\mathrm{p}$-value & $\beta$ & SE & $p$-value \\
\hline GLS at baseline & -0.46 & 0.07 & $<0.001$ & - & - & - \\
\hline GCS at baseline & - & - & - & -0.46 & 0.07 & $<0.001$ \\
\hline$\Delta \mathrm{rAlx}$ & -0.11 & 0.07 & 0.10 & -0.13 & 0.07 & 0.057 \\
\hline$\triangle V A C$ & -0.15 & 0.07 & 0.026 & 0.04 & 0.07 & 0.53 \\
\hline$\triangle P W V$ & -0.10 & 0.07 & 0.13 & 0.08 & 0.07 & 0.25 \\
\hline$\triangle$ Mean 24-hour SBP & 0.02 & 0.07 & 0.72 & 0.03 & 0.07 & 0.70 \\
\hline$\triangle \mathrm{DDD}$ & -0.003 & 0.07 & 0.97 & 0.05 & 0.07 & 0.53 \\
\hline
\end{tabular}

$\triangle$ - change at 12-month follow-up (follow-up value minus baseline value); GLS - global longitudinal strain; GCS - global circumferential strain; CAP HR 75 - central augmentation pressure at heart rate 75 beats/min; CAGPH HR 75 - central augmentation index at heart rate 75 beats/min; VAC - ventricular-arterial coupling; PWV - pulse wave velocity; SBP - systolic blood pressure; DDD - daily defined dose of hypertension drugs; rAlx - radial augmentation index. Values in bold are statistically significant.

Table 7. Sensitivity analysis

\begin{tabular}{|c|c|c|c|c|c|c|c|}
\hline \multirow[b]{2}{*}{ Variables } & \multirow{2}{*}{$\begin{array}{l}\text { GLS deterioration } \\
\text { at follow-up } \\
n=72\end{array}$} & \multirow{2}{*}{$\begin{array}{l}\text { GLS improvement } \\
\begin{array}{c}\text { at follow-up } \\
n=172\end{array}\end{array}$} & \multirow[b]{2}{*}{$\mathrm{p}$-value } & \multicolumn{2}{|c|}{$\Delta \mathrm{GLS}$} & \multicolumn{2}{|c|}{$\triangle \mathrm{GCS}$} \\
\hline & & & & r & $p$-value & $r$ & $\mathrm{p}$-value \\
\hline$\triangle P W V$ & $0.33 \pm 1.75$ & $-0.23 \pm 2.18$ & 0.10 & -0.14 & 0.11 & 0.01 & 0.88 \\
\hline$\triangle \mathrm{CAGPH} H \mathrm{R} 75$ [\%] & $1.29 \pm 7.79$ & $-1.16 \pm 5.97$ & 0.048 & -0.19 & 0.029 & -0.20 & 0.035 \\
\hline$\triangle \mathrm{CAP} H \mathrm{R} 75[\mathrm{~mm} \mathrm{Hg}]$ & $0.71 \pm 4.65$ & $-1.06 \pm 3.60$ & 0.017 & -0.25 & 0.004 & -0.27 & 0.004 \\
\hline$\triangle \mathrm{VAC}$ & $0.08 \pm 0.30$ & $-0.02 \pm 0.23$ & 0.034 & -0.22 & 0.010 & -0.02 & 0.81 \\
\hline
\end{tabular}

GLS - global longitudinal strain; $\triangle$ - change at 12-month follow-up (follow-up value minus baseline value); GCS - global circumferential strain; PWV - pulse wave velocity; CAGPH HR 75 - central augmentation index at heart rate 75 beats/min; CAP HR 75 - central augmentation pressure at heart rate 75 beats/min; VAC - ventricular-arterial coupling. Values in bold are statistically significant.

$r=0.96$ ); therefore, we would expect similar associations of changes in both central and radial augmentation index with changes in GLS and GCS. These associations were observed in the current study, which highlights the potential value of using non-transformed peripheral waveform parameters as risk stratification tools to supplement derived central BP measures that may be subject to error from cuff calibration and generalized transfer functions. ${ }^{33-35}$

The link between LV load and myocardial function is well-documented. ${ }^{36}$ The value of LV multidirectional deformation results from loading conditions and myocardial determinants, including passive tissue characteristics, crossover of myofibers in the subendocardial and subepicardial layers, and direction of myofibers. Impairment in GLS represents the first step in the cascade of LV remodeling in the natural history of $\mathrm{HHD}$, and may parallel or even precede other clinical forms of myocardial affection such as LV hypertrophy, diastolic dysfunction, or left atrial structural and functional abnormalities. ${ }^{37-42}$ Conversely, LV circumferential performance deteriorates in more advanced stages of the disease, and may even increase the asymptomatic phase of heart failure (stage B) to compensate for the dysfunction in the longitudinal direction. ${ }^{10,43-48}$
This paper demonstrated that changes in LV longitudinal systolic deformation over follow-up were associated with alterations in relevant LV afterload-associated indices, among which central augmentation pressure remained an independent predictor after multivariable adjustments for possible confounders. Similar to GLS, the independent contribution from central augmentation pressure parameters was evident for LV circumferential strain as well. Moreover, we discovered that the interaction between the left ventricle and arterial system as assessed by VAC, representing net cardiovascular performance, independently determined changes in LV longitudinal strain. Importantly, our results demonstrated superiority of comprehensive evaluation of LV afterload, using non-invasive hemodynamic parameters derived from PWA over cuff BP measurement, in the context of LV function changes during antihypertensive therapy.

A stronger association between hypertension-related abnormalities of myocardial performance with pulse wave parameters rather than with brachial BP has been reported in previous papers; ${ }^{17,49-51}$ however, a lack of research currently exists regarding the analogous interaction with respect to changes in LV strain over time. Accordingly, our analysis expounds upon prior reports by using repeated observations at the individual level, which may support 
a causal nature of demonstrated relationships. However, as this study is retrospective, a post hoc analysis of the results from a randomized clinical trial should be regarded as hypothesis-generating. Despite the different trajectories in the early phase of HHD, both GLS and GCS are prone to the adverse influence of LV afterload. Thus, the extension of diagnostic focus over these 2 components of LV systolic function from the beginning of patient follow-up may be reasonable to track myocardial pathology in order to assist in therapeutic decision-making. Recognition for the role of hemodynamic loading factors and myocardial deformation, especially GLS - a widely recognized prognosticator in patient management strategy, might provide further clinical benefits. Our results provisionally support the concept of using non-invasive large artery hemodynamic assessment as a clinical rather than a research-only tool.

\section{Limitations}

This study population profile encompassing low to medium risk subjects with uncomplicated HT constrains the ability to extrapolate the results to other cohorts, especially with severe HT or advanced HHD. The estimations of central hemodynamics in the current analysis were acquired noninvasively and using a calibration, based on brachial cuff systolic and DBP. Thus, the readings will not be the same as invasively measured BP waveforms.

\section{Conclusions}

In hypertensive patients, LV longitudinal and circumferential functional remodeling over time is associated with arterial hemodynamic characteristics. This may indicate the need for pulse wave analysis to optimize patient care.

\section{ORCID iDs}

Anna Teresa Goździk (1) https://orcid.org/0000-0002-8182-5586 Ewelina Jasic-Szpak (1) https://orcid.org/0000-0002-4976-2589 Jakub Michałowicz (10) https://orcid.org/0000-0002-4940-4108 Monika Przewłocka-Kosmala (1) https://orcid.org/0000-0002-4054-6521 James Edward Sharman (10 https://orcid.org/0000-0003-2792-0811 Wojciech Kosmala (10 https://orcid.org/0000-0003-3807-8201

\section{References}

1. Fung MJ, Thomas L, Leung DY. Left ventricular function and contractile reserve in patients with hypertension. Eur Heart J Cardiovasc Imaging. 2018;19(11):1253-1259. doi:10.1093/ehjci/jex338

2. Lee WH, Liu YW, Yang LT, Tsai WC. Prognostic value of longitudinal strain of subepicardial myocardium in patients with hypertension. J Hypertens. 2016;34(6):1195-1200. doi:10.1097/HJH.000000000 0000903

3. Modin D, Biering-Sorensen SR, Mogelvang R, LandlerN, Jensen JS, BieringSorensen T. Prognostic value of echocardiography in hypertensive versus nonhypertensive participants from the general population. Hypertension. 2018;71(4):742-751. doi:10.1161/HYPERTENSIONAHA. 117.10674

4. Cheng S, McCabe EL, Larson MG, et al. Distinct aspects of left ventricular mechanical function are differentially associated with cardiovascular outcomes and all-cause mortality in the community. J Am Heart Assoc. 2015;4(10):e002071. doi:10.1161/JAHA.115.002071
5. Mancusi C, Losi MA, Izzo R, et al. Higher pulse pressure and risk for cardiovascular events in patients with essential hypertension: The Campania Salute Network. Eur JPrev Cardiol. 2018;25(3):235-243. doi:10.1177/2047487317747498

6. Galderisi M, Trimarco B. Global longitudinal strain: A novel hallmark of cardiac risk in arterial hypertension. J Hypertens. 2016;34(6):1050-1051. doi:10.1097/HJH.0000000000000920

7. de Simone G, Mancusi C, Esposito R, De Luca N, Galderisi M. Echocardiography in arterial hypertension. High Blood Press Cardiovasc Prev. 2018;25(2):159-166. doi:10.1007/s40292-018-0259-y

8. Shin SM, Shim WJ, Park SM. Early changes of left ventricular function in young adults with never-treated hypertension and no left ventricular hypertrophy: Relationships to ambulatory blood pressure monitoring. Clin Exper Hypertens. 2014;36(7):517-523. doi:10.3109/ 10641963.2013.863326

9. Sun JP, Xu T, Yang Y, et al. Layer-specific quantification of myocardial deformation may disclose the subclinical systolic dysfunction and the mechanism of preserved ejection fraction in patients with hypertension. Int J Cardiol. 2016;219:172-176. doi:10.1016/j.ijcard. 2016.06.035

10. Ballo P, Nistri S, Cameli M, et al. Association of left ventricular longitudinal and circumferential systolic dysfunction with diastolic function in hypertension: A nonlinear analysis focused on the interplay with left ventricular geometry. J Card Fail. 2014;20(2):110-120. doi:10.1016/j. cardfail.2013.12.009

11. Kraigher-Krainer E, Shah AM, Gupta DK, et al. Impaired systolic function by strain imaging in heart failure with preserved ejection fraction. J Am Coll Cardiol. 2014;63(5):447-456. doi:10.1016/j.jacc.2013.09.052

12. Kaess BM, Rong J, Larson MG, et al. Relations of central hemodynamics and aortic stiffness with left ventricular structure and function: The Framingham Heart Study. J Am Heart Assoc. 2016;5(3):e002693. doi:10.1161/JAHA.115.002693

13. Kaess BM, Rong J, Larson MG, et al. Aortic stiffness, blood pressure progression, and incident hypertension. JAMA. 2012;308(9):875-881. doi:10.1001/2012.jama.10503

14. Russo C, Jin Z, Takei Y, et al. Arterial wave reflection and subclinical left ventricular systolic dysfunction.J Hypertens. 2011;29(3):574-582. doi:10.1097/HJH.0b013e328342ca56

15. Russo C, Jin Z, Palmieri V, et al. Arterial stiffness and wave reflection: Sex differences and relationship with left ventricular diastolic function. Hypertension. 2012;60(2):362-368. doi:10.1161/HYPERTENSIONAHA. 112.191148

16. Kim HL, Seo JB, Chung WY, Kim SH, Kim MA, Zo JH. Independent association between brachial-ankle pulse wave velocity and global longitudinal strain of left ventricle. Int J Cardiovasc Imaging. 2015;31(8): 1563-1570. doi:10.1007/s10554-015-0744-5

17. Ye Z, Coutinho T, Pellikka PA, Villarraga HR, Borlaug BA, Kullo IJ. Associations of alterations in pulsatile arterial load with left ventricular longitudinal strain. Am J Hypertens. 2015;28(11):1325-1331. doi:10. 1093/ajh/hpv039

18. Krishnasamy R, Hawley CM, Stanton T, et al. Left ventricular global longitudinal strain is associated with cardiovascular risk factors and arterial stiffness in chronic kidney disease. BMCNephrol. 2015;16:106. doi:10.1186/s12882-015-0098-1

19. Negishi K, Yang H, Wang Y, et al. Importance of calibration method in central blood pressure for cardiac structural abnormalities. Am J Hypertens. 2016;29(9):1070-1076. doi:10.1093/ajh/hpw039

20. Protogerou AD, Argyris AA, Papaioannou TG, et al. Left-ventricular hypertrophy is associated better with 24-h aortic pressure than 24-h brachial pressure in hypertensive patients: The SAFAR study.J Hypertens. 2014;32(9):1805-1814. doi:10.1097/HJH.0000000000000263

21. Zhang $Y$, Kollias $G$, Argyris AA, et al. Association of left ventricular diastolic dysfunction with 24-h aortic ambulatory blood pressure: The SAFAR study. J Hum Hypertens. 2015;29(7):442-448. doi:10.1038/ jhh.2014.101

22. Sharman JE, Marwick TH, Abhayaratna WP, Stowasser M. Rationale and design of a randomized study to determine the value of central Blood Pressure for GUIDing managEment of hypertension: The BP GUIDE study. Am Heart J. 2012;163(5):761-767. doi:10.1016/j.ahj.2012.02.017

23. WHO Collaborating Centre for Drug Statistics Methodology. Norwegian Institute of Public Health. Daily Defined Dose: Definition and General Considerations. www.whocc.no/ddd/definition_and_general_ considera. Accessed January 10, 2019. 
24. Lang RM, Bierig M, Devereux RB, et al. Recommendations for cardiac chamber quantification by echocardiography in adults: An update from the American Society of Echocardiography and the European Association of Cardiovascular Imaging. Eur Heart J Cardiovasc Imaging. 2015;16(3):233-271. doi:10.1093/ehjci/jev014

25. Nagueh SF, Smiseth OA, Appleton CP, et al. Recommendation for the evaluation of left ventricular diastolic function by echocardiography: An update from the American Society of Echocardiography and the European Association of Cardiovascular Imaging. J Am SoC Echocardiogr. 2016;29(4):277-314. doi:10.1016/j.echo.2016.01.011

26. Sharman JE, Avolio AP, Baulmann J, et al. Validation of non-invasive central blood pressure devices: ARTERY Society task force consensus statement on protocol standardization. Eur Heart J. 2017;38(37): 2805-2812. doi:10.1093/eurheartj/ehw632

27. Chen $\mathrm{CH}$, Fetics $\mathrm{B}$, Nevo E, et al. Noninvasive single-beat determination of left ventricular endsystolic elastance in humans. J Am Coll Cardiol. 2001;38(7):2028-2034. doi:10.1016/s0735-1097(01)01651-5

28. Kollias A, Lagou S, Zeniodi ME, Boubouchairpoulou N, Sterigou GS. Association of central versus brachial blood pressure with TargetOrgan Damage: Systematic review and meta-analysis. Hypertension. 2016;67(1):183-190. doi:10.1161/HYPERTENSIONAHA.115.06066

29. Sharman JE, Laurent S. Central blood pressure in the management of hypertension: Soon reaching the goal? J Hum Hypertens. 2013; 27(7):405-411. doi:10.1038/jhh.2013.23

30. Armstrong MK, Schultz MG, Picone DS, Sharman JE. Aortic to brachial artery stiffness gradient is not blood pressure independent. J Hum Hypertens. 2019;33(5):385-392. doi:10.1038/s41371-018-0154-y

31. Climie RE, Schultz MG, Fell JW, Romero L, Otahal P, Sharman JE. Central to brachial blood pressure amplification in type 2 diabetes: A systematic review and meta-analysis. J Hum Hypertens. 2019;33(2):94-105. doi:10.1038/s41371-018-0124-4

32. Millasseau SC, Patel SJ, Redwood SR, Ritter JM, Chowienczyk PJ. Pressure wave reflection assessed from the peripheral pulse: Is a transfer function necessary? Hypertension. 2003;41(5):1016-1020. doi:10. 1161/01.HYP.0000057574.64076.A5

33. Picone DS, Schultz MG, Otahal $P$, et al. Accuracy of cuff-measured blood pressure: Systematic reviews and meta-analyses. J Am Coll Cardiol. 2017;70(5):572-586. doi:10.1016/j.jacc.2017.05.064

34. Schultz MG, Picone DS, Armstrong MK, et al. Validation study to determine the accuracy of central blood pressure measurement using the sphygmocor xcel cuff device. Hypertension. 2020;76(1):244-250. doi:10.1161/HYPERTENSIONAHA.120.14916

35. Picone DS, Schultz MG, Peng X, et al. Intra-arterial analysis of the best calibration methods to estimate aortic blood pressure. $J$ Hypertens. 2019;37(2):307-315. doi:10.1097/HJH.0000000000001902

36. Kosmala W, Przewlocka-Kosmala M, Sharman JE, Schultz MG, Marwick TH. Stability of left ventricular longitudinal and circumferential deformation over time and standard loading conditions. Eur Heart J Cardiovasc Imaging. 2017;18(9):1001-1007. doi:10.1093/ehjci/jew135

37. Kosmala W, Plaksej R, Strotmann JM, et al. Progression of left ventricular functional abnormalities in hypertension patients with heart failure: An ultrasonic two dimensional speckle tracking study. J Am SoC Echocardiogr. 2008;21(12):1309-1317. doi:10.1016/j.echo.2008.10.006
38. Lembo M, Esposito R, Li F, et al. Impact of pulse pressure on left ventricular global longitudinal strain in normotensive and newly diagnosed, untreated hypertensive patients. J Hypertens. 2016;34(6): 1201-1207. doi:10.1097/HJH.0000000000000906

39. Przewlocka-Kosmala M, Jasic-Szpak E, Rojek A, Kabaj M, Sharman JE, Kosmala W. Association of central blood pressure with left atrial structural and functional abnormalities in hypertensive patients: Implications for atrial fibrillation prevention. Eur J Prev Cardiol. 2019; 26(10):1018-1027. doi:10.1177/2047487319839162

40. Szelenyi Z, Fazakas A, Szenasi G, et al. The mechanism of reduced longitudinal left ventricular systolic function in hypertensive patients with normal ejection fraction. J Hypertens. 2015;33(9):1962-1969. doi:10.1097/HJH.0000000000000624

41. Kosmala W, Marwick TH, Stanton T, Abhayaratna WP, Stowasser M, Sharman JE. Guiding hypertension management using central blood pressure: Effect of medication withdrawal on left ventricular function. Am J Hypertens. 2016;29(3):319-325. doi:10.1093/ajh/hpv108

42. Huang $H$, Ruan $Q$, Lin M, Yan L, Huang Ch, Fu L. Investigation on left ventricular multidirectional deformation in patients of hypertension with different LVEF. Cardiovascular Ultrasound. 2017;15(1):14. doi:10. 1186/s12947-017-0106-7

43. Russo C, Jin Z, Elkind MS, et al. Prevalence and prognostic value of subclinical left ventricular systolic dysfunction by global longitudinal strain in a community-based cohort. Eur J Heart Fail. 2014;16(12): 1301-1309. doi:10.1002/ejhf.154

44. Tadic M, Majstorovic A, Pencic B, et al. The impact of high-normal blood pressure on left ventricular mechanics: A three-dimensional and speckle tracking echocardiography study. Int J Cardiovasc Imaging. 2014;30(4):699-711. doi:10.1007/s10554-014-0382-3

45. Tadic M, Cuspidi C. Left ventricular strain and arterial hypertension: Is longitudinal strain ready for primetime? JClin Hypertens. 2020;22(4): 683-685. doi:10.1111/jch.13833

46. Przewlocka-Kosmala M, Kosmala W, Mazurek W. Left ventricular circumferential function in patients with essential hypertension. J Hum Hypertens. 2006;20(9):666-671. doi:10.1038/sj.jhh.1002054

47. Morris DA, Otani $K$, Bekfani T, et al. Multidirectional global left ventricular systolic function in normal subjects and patients with hypertension: Multicenter evaluation. J Am Soc Echocardiogr. 2014;27(5): 493-500. doi:10.1016/j.echo.2014.01.017

48. Kim D, Shim CHY, Hong GR, et al. Differences in left ventricular functional adaptation to arterial stiffness and neurohormonal activation in patients with hypertension: A study with two-dimensional layer-specific speckle tracking echocardiography. Clin Hypertens. 2017;23:21. doi:10.1186/s40885-017-0078-9

49. Hwang JW, Kang SJ, Lim HS, et al. Impact of arterial stiffness on regional myocardial function assessed by speckle tracking echocardiography in patients with hypertension. J Cardiovasc Ultrasound. 2012;20(2):90-96. doi:10.4250/jcu.2012.20.2.90

50. Kim HL, Lim WH, Jae-Bin Seo JB, et al. Association between arterial stiffness and left ventricular diastolic function in relation to gender and age. Medicine (Baltimore). 2017;96(1):e5783. doi:10.1097/MD. 0000000000005783

51. Krzesiński P, Uziębło-Życzkowska B, Gielerak G, Stańczyk A, Kurpaska M, Piotrowicz K. Global longitudinal two-dimensional systolic strain is associated with hemodynamic alterations in arterial hypertension. J Am Soc Hypertens. 2015;9(9):680-689. doi:10.1016/j.jash.2015.06.014 